\title{
Tipos polínicos encontrados em amostras de méis de Apis mellifera em Picos, Estado do Piauí
}

\author{
Pollinic types found in honey samples of Apis mellifera from Picos, State of Piaui
}

\author{
Geni da Silva Sodré ${ }^{\mathrm{I}}$ Luís Carlos Marchini ${ }^{\mathrm{II}}$ Augusta Carolina de Camargo Carmelo Moreti ${ }^{\mathrm{II}}$ \\ Carlos Alfredo Lopes de Carvalho ${ }^{\mathrm{I}}$
}

\begin{abstract}
Com o objetivo de determinar os tipos polínicos
roduzidos por Apis mellifera L., 1758, no município RESUMO de méis produzidos por Apis mellifera L., 1758, no município
de Picos, Estado do Piauí, foram realizadas análises polínicas de 35 amostras de méis coletadas entre novembro e dezembro de 2001 no Laboratório de Apicultura do Departamento de Entomologia, Fitopatologia e Zoologia Agrícola da Escola Superior de Agricultura "Luiz de Queiroz”, Universidade de São Paulo. A identificação dos tipos polínicos foi realizada por meio de descrições obtidas em literatura especializada. Os resultados demonstraram que foram encontrados 36 tipos polínicos, distribuídos em 18 famílias botânicas, sendo consideradas como pólen dominante Piptadenia sp. (Mimosaceae), Mimosa caesalpiniiaefolia Benth. (Mimosaceae), M. verrucosa Benth. (Mimosaceae) e Croton urucurana Baill. (Euphorbiaceae).
\end{abstract}

Palavras-chave: mel, pólen, Apis mellifera L., Mimosaceae, Piptadenia.

\section{ABSTRACT}

This research deals with the pollinic types of honeys produced by Apis mellifera L., 1758 in the municipality of Picos, State of Piaui were determined in the Laboratory of Apiculture, Department of Entomology, Phytopathology and Agricultural Zoology, College of Agriculture 'Luiz de Queiroz', University of São Paulo, in Piracicaba, State of São Paulo, pollinic analysis of 36 honey samples collected in November and December of 2001. The identification of e pthollinic made by types was specialized literature. The results showed that 36 types of pollen were found, distributed in 18 botanical families, and the following plant species were considered as dominant Piptadenia sp. (Mimosaceae), Mimosa caesalpiniiaefolia Benth.
(Mimosaceae), M. verrucosa Benth. (Mimosaceae) and Croton urucurana Baill. (Euphorbiaceae).

Key words: honey, pollen, Apis mellifera L., Mimosaceae, Piptadenia.

O Estado do Piauí tem sido destaque nacional quanto ao potencial apícola por possuir diversas formações vegetais (Caatinga, Cerrado, Floresta semi-decidual, Restinga e Mangue) com boas características para a apicultura, temperatura elevada, umidade relativa do ar em torno de $70 \%$, e boa luminosidade com floradas ricas e variadas (SILVA et al., 2002). De acordo com esses autores, o Estado dispõe de uma vegetação arbórea, herbácea e arbustiva abundante e bem distribuída. Entretanto, há poucas informações sobre a flora apícola.

O conhecimento da flora apícola é importante para identificar as espécies vegetais utilizadas pelas abelhas. Essas espécies assumem grande importância por indicar aos apicultores fontes adequadas e de abundante suprimento de néctar e pólen, contribuindo para a formação do mel produzido na região. O conhecimento da flora apícola também é necessário para a preservação e a multiplicação destas plantas de potencial melífero, auxiliando o estabelecimento de uma apicultura sustentável (HOWER, 1953; MORETI et al., 1998; SANTOS et al., 2002).

\footnotetext{
ICentro de Ciências Agrárias, Ambientais e Biológica, Universidade Federal do Recôncavo da Bahia (UFRB), Grupo de Pesquisa Insecta, 44380-000, Cruz das Almas, BA, Brasil. E-mail: gssodre@yahoo.com.br. *Autor para correspôndência.

"Departamento de Entomologia, Fitopatologia e Zoologia Agrícola, Escola Superior de Agricultura "Luiz de Queiroz” (ESALQ), Universidade de São Paulo (USP), Piracicaba, SP, Brasil.

IIIInstituto de Zootecnia, Agência Paulista de Tecnologia dos Agronegócios (SSA), Nova Odessa, SP, Brasil.
} 
A tipificação do grão de pólen de amostras de méis é de grande importância no controle de qualidade desse alimento, pois torna possível atestar sua procedência e detectar adulterações (SANTOS JÚNIOR \& SANTOS, 2002). O pólen coletado involuntariamente pelas abelhas no momento da coleta do néctar, tornando-se presente no mel elaborado, constitui importante indicador de sua origem botânica e, principalmente, geográfica. A análise quantitativa de grãos de pólen permitirá estabelecer a proporção que cada planta nectarífera contribui para a constituição do mel (IWAMA \& MELHEN, 1979; BARTH, 1989; BASTOS, 2002).

A origem botânica dos méis é caracterizada por sua análise microscópica, especialmente a identificação e a contagem de grãos de pólen. O método é baseado na identificação do grão de pólen pela avaliação microscópica comparando-os aos tipos polínicos descritos na literatura (D’ALBORE \& PERSANO-ODDO, 1978; MOORE \& WEBB, 1978; SAWYER \& PICKARD, 1988).

No Estado do Piauí, o município de Picos vem se destacando pelo elevado potencial na produção de mel de excelente qualidade. Desse modo, o presente estudo teve como objetivo conhecer os tipos polínicos encontrados em amostras de méis de Apis mellifera L., 1758 (Hymenoptera: Apidae) desse município, indicando os recursos tráficos utilizados pelas abelhas e contribuindo para a identificação das plantas apícolas do Estado.

As amostras de méis produzidos por Apis mellifera foram coletadas no período de novembro a dezembro de 2001, diretamente de apicultores do município de Picos, Estado do Piauí, totalizando 35 amostras.

As análises polínicas foram realizadas no Laboratório de Apicultura do Departamento de Entomologia, Fitopatologia e Zoologia Agrícola da Escola Superior de Agricultura "Luiz de Queiroz", campus de Piracicaba, Universidade de São Paulo.

Todas as amostras de méis foram preparadas utilizando-se o método da acetólise (ERDTMAN, 1952) e analisadas por dois métodos: a) método qualitativo - os tipos polínicos presentes nos méis foram determinados por comparação com o laminário referência e as descrições obtidas em literatura especializada (BARTH, 1970 a,b,c, 1971a,b, 1989, 1990) e b) método quantitativo - foi realizada a contagem consecutiva de 300 grãos de pólen/ repetição/amostra determinando-se as classes de ocorrência, que segundo LOUVEAUX et al. (1978), são: pólen dominante (PD - > 45\% do total de grãos), pólen acessório (PA-16 a 45\%), pólen isolado importante (PII - 3 a 15\%) e pólen isolado ocasional (PIO - <3\%). Os resultados apresentados são as médias de duas repetições.

A análise polínica qualitativa das amostras de méis demonstrou uma grande diversidade de fontes de néctar visitada por A. mellifera em Picos-PI. Foram encontrados 36 tipos polínicos, distribuídos em 18 famílias botânicas (Tabela 1).

Os tipos polínicos dominantes nas amostras foram: Piptadenia sp. (Mimosaceae) (77,22\%), $\boldsymbol{M}$. caesalpiniiaefolia (Mimosaceae) (2,86\%), $\boldsymbol{M}$. verrucosa (Mimosaceae) (2,86\%) e C. urucurana (Euphorbiaceae) (2,86\%) (Tabela 1).

A análise polínica quantitativa das amostras de méis demonstrou a importante participação do tipo Piptadenia sp. (Mimosaceae), reiterando o potencial apícola deste gênero no município de procedência das amostras. No presente trabalho, foi constatada a presença deste gênero como tipo dominante em 27 das 35 amostras avaliadas. As espécies $\boldsymbol{M}$. caesalpiniiaefolia, $M$. verrucosa e $C$. urucurana apareceram como pólen dominante em apenas uma das 35 amostras avaliadas. De acordo com BARTH (1989), quando predomina nas amostras de méis o grão de pólen de Mimosaceae do tipo Piptadenia moniliformis, isso pode indicar que as amostras são originárias do Estado do Piauí.

A ocorrência de tipos polínicos acessórios nas amostras foi elevada (45,71\% do total das amostras). De acordo com BARTH (1970b), os méis com pólen acessório são muito freqüentes em função da atividade apícola brasileira ainda ser realizada de maneira rudimentar quanto ao pasto apícola. Neste estudo, foram observadas cinco amostras com pólen acessório sem a presença de pólen dominante. Os tipos polínicos acessórios foram Chenopodium sp., $\boldsymbol{C}$. urucurana, Sida sp., Tibouchina sp., $M$. caesalpiniiaefolia, M. verrucosa, Piptadenia sp., Tipo Myrcia, Borreria sp. 1 e Richardia sp. (Tabela 1).

Quanto ao pólen isolado importante e isolado ocasional, pode ser observada sua grande freqüência (Tabela 1). Esses polens têm pouca importância quanto à quantidade de néctar fornecido, entretanto, eles fornecem informação quanto à origem e procedência geográfica da amostra (BARTH, 1989).

Os méis característicos do município de Picos, Estado do Piauí, contêm como principal pólen dominante o tipo Piptadenia sp., cujo gênero merece atenção especial por parte dos apicultores locais, tanto no sentido de sua preservação, quanto no sentido de sua multiplicação para ampliação do pasto apícola. 
Tabela 1 - Porcentagem de tipos polínicos presentes em 35 amostras de méis de Apis mellifera do município de Picos, Estado do Piauí.

\begin{tabular}{|c|c|c|c|c|c|}
\hline \multirow[t]{2}{*}{ Família } & \multirow[t]{2}{*}{ Tipos polínicos } & \multicolumn{4}{|c|}{ Porcentagem de classe de ocorrência* } \\
\hline & & PD & PA & PII & $\mathrm{PIO}$ \\
\hline Amaranthaceae & Alternanthera ficoidea L. & 0,00 & 0,00 & 0,00 & 2,86 \\
\hline Arecaceae & Tipo Arecaceae & 0,00 & 0,00 & 2,86 & 0,00 \\
\hline \multirow[t]{3}{*}{ Asteraceae } & Bidens sp. & 0,00 & 0,00 & 2,86 & 14,28 \\
\hline & Emilia sp. & 0,00 & 0,00 & 0,00 & 5,71 \\
\hline & Mikania sp. & 0,00 & 0,00 & 5,71 & 5,71 \\
\hline Caesalpiniaceae & Tipo Caesalpiniaceae & 0,00 & 0,00 & 2,86 & 2,86 \\
\hline Chenopodiaceae & Chenopodium sp. & 0,00 & 2,86 & 14,28 & 51,42 \\
\hline \multirow[t]{2}{*}{ Compositae } & Vernonia cognata Less. & 0,00 & 0,00 & 2,86 & 2,86 \\
\hline & Vernonia sp. & 0,00 & 0,00 & 0,00 & 2,86 \\
\hline \multirow[t]{2}{*}{ Euphorbiaceae } & Croton urucurana Baillon. & 2,86 & 2,86 & 11,43 & 22,85 \\
\hline & Croton sp. & 0,00 & 0,00 & 2,86 & 2,86 \\
\hline \multirow[t]{3}{*}{ Fabaceae } & Bauhinia sp. & 0,00 & 0,00 & 5,71 & 0,00 \\
\hline & Schrankia sp. & 0,00 & 0,00 & 2,86 & 5,71 \\
\hline & Tipo Fabaceae & 0,00 & 0,00 & 11,43 & 5,71 \\
\hline \multirow[t]{2}{*}{ Lamiaceae } & Hyptis eriophylla Pohl ex Benth. & 0,00 & 0,00 & 2,86 & 14,28 \\
\hline & Salvia sp. & 0,00 & 0,00 & 2,86 & 2,86 \\
\hline Malvaceae & Sida sp. & 0,00 & 2,86 & 5,71 & 25,74 \\
\hline Melastomataceae & Tibouchina sp. & 0,00 & 34,31 & 25,72 & 22,86 \\
\hline \multirow[t]{5}{*}{ Mimosaceae } & Leucaena leucocephala Lam. & 0,00 & 0,00 & 0,00 & 2,86 \\
\hline & Mimosa caesalpinifolia Benth. & 2,86 & 8,58 & 17,16 & 17,16 \\
\hline & Mimosa scabrella Benth. & 0,00 & 0,00 & 0,00 & 14,28 \\
\hline & Mimosa verrucosa Benth. & 2,86 & 2,86 & 11,43 & 22,85 \\
\hline & Piptadenia sp. & 77,22 & 5,71 & 8,58 & 2,86 \\
\hline \multirow[t]{2}{*}{ Myrtaceae } & Eucalyptus sp. & 0,00 & 0,00 & 0,00 & 2,86 \\
\hline & Tipo Myrcia & 0,00 & 2,86 & 17,16 & 5,71 \\
\hline \multirow[t]{3}{*}{ Poaceae } & Brachiaria decumbens Stapf. & 0,00 & 0,00 & 2,86 & 0,00 \\
\hline & Paspalum notatum Fluegg & 0,00 & 0,00 & 0,00 & 2,86 \\
\hline & Zea mays L. & 0,00 & 0,00 & 0,00 & 2,86 \\
\hline \multirow[t]{4}{*}{ Rubiaceae } & Borreria sp. 1 & 0,00 & 2,86 & 22,85 & 25,73 \\
\hline & Borreria sp. 2 & 0,00 & 0,00 & 17,16 & 8,58 \\
\hline & Richardia sp. & 0,00 & 2,86 & 11,43 & 14,28 \\
\hline & Tipo Rubiaceae & 0,00 & 0,00 & 0,00 & 2,86 \\
\hline Rutaceae & Citrus sp. & 0,00 & 0,00 & 0,00 & 2,86 \\
\hline Sapindaceae & Serjania sp. & 0,00 & 0,00 & 0,00 & 14,28 \\
\hline Solanaceae & Tipo Solanaceae & 0,00 & 0,00 & 0,00 & 2,86 \\
\hline Não determinado & & 0,00 & 0,00 & 0,00 & 2,86 \\
\hline
\end{tabular}

*PD = pólen dominante maior que 45\% do total de grão de pólen, PA = pólen acessório de 16 a 45\%, PII = pólen isolado importante de 3 a $15 \%$ e PIO = pólen isolado ocasional menor que $3 \%$.

\section{AGRADECIMENTOS}

À Fundação de Amparo à Pesquisa do Estado da Bahia (FAPESB) pelas concessões da bolsa de Pós-doutorado para a autora Geni da Silva Sodré e ao Conselho Nacional de Desenvolvimento Científico e Tecnológico (CNPq), pela concessão da bolsa de Produtividade em Pesquisa para o autor Carlos Alberto Lopes de Carvalho.

\section{REFERÊNCIAS}

BARTH, O.M. Análise microscópica de algumas amostras de mel. 1. Pólen dominante. Anais da Academia Brasileira de Ciências, v.42, p.351-366, 1970a.
BARTH, O.M. Análise microscópica de algumas amostras de mel. 2. Pólen Acessório. Anais da Academia Brasileira de Ciências, v.42, p.571-590, 1970b.

BARTH, O.M. Análise microscópica de algumas amostras de mel. 3. Pólen Isolado. Anais da Academia Brasileira de Ciências, v.42, p.747-772, 1970c.

BARTH, O.M. Análise microscópica de algumas amostras de mel. 6. Espectro polínico de algumas amostras de mel dos Estados da Bahia e do Ceará. Revista Brasileira de Biologia, v.31, p.431-434, 1971a.

BARTH, O.M. Mikroskopische bestandteile brasilianischer honigtauhonige. Apidologie, v.2, p.157-167, 1971b. 
BARTH, O.M. O pólen no mel brasileiro. Rio de Janeiro: Luxor, 1989. 226p.

BARTH, O.M. Pollen in monofloral honeys from Brazil. Journal of Apicultural Research, v.29, p.89-94, 1990.

BASTOS, E.M.A.F. Origem botânica do mel e da própolis produzidos por abelhas, determinados por observações em campo, métodos microscópicos e RAPD. In: CONGRESSO BAIANO DE APICULTURA, 2002, Salvador, Ba. Anais... Salvador: UESC, 2002. p.32-33.

D’ALBORE, G.R.; PERSANO-ODDO, L. Flora apistica italiana. Milano: Federazione Apicoltori Italiani, 1978. 51p.

ERDTMAN, G. Pollen morphology and plant taxonomy Angiosperms. Stockholm: Almqvist \& Wiksell, 1952. 539p.

HOWER, F.N. Plantas melíferas. Barcelona: Reverté, 1953. 35p.

IWAMA, S.; MELHEM, T.S. The pollen spectrum of the honey of Tetragonisca angustula angustula Latrelle (Apidae, Meliponinae). Apidologie, v.10, n.3, p.275-295, 1979.

LOUVEAUX, J. et al. Methods of Melissopalinology. Bee World, v.59, n.4, p.139-157, 1978.
MOORE, P.D.; WEBB, J.A. Anales illustrated guide to pollen analysis. London: Hodder and Stoughton, 1978. 133p.

MORETI, A.C. de C.C. et al. Caracterização das plantas apícolas do Centro de Apicultura Tropical / Instituto de Zootecnia, em Pindamonhangaba, SP. In: CONGRESSO BRASILEIRO DE APICUlTURA, 12., 1998, Salvador, Ba. Anais... Salvador: Confederação Brasileira de Apicultura, 1998. p.179.

SANTOS, N.C.R. et al. Plantas visitadas por Apis mellifera L. no município de Alagoinhas-BA: dados preliminares. In: CONGRESSO NACIONAL DE BOTÂNICA, 53.; REUNIÃO NORDESTINA DE BOTÂNICA, 25., 2002, Recife, Pe. Resumos... Recife: Sociedade Botânica do Brasil, 2002. p.261.

SANTOS JÚNIOR, M.C.; SANTOS, F.A.R. Identificação botânica de méis da Bahia: estudo palinológico. In: CONGRESSO NACIONAL DE BOTÂNICA, 53.; REUNIÃO NORDESTINA DE BOTÂNICA, 25., 2002, Recife, Pe. Resumos... Recife: Sociedade Botânica do Brasil, 2002. p.191.

SAWYER, R.M.; PICKARD, R.S. Honey identification. Cardiff: Academic, 1988. 115p.

SILVA, A.F. da et al. Zoneamento apibotânico de ecossistemas do Piauí (Resultados preliminares). In: CONGRESSO BRASILEIRO DE APICULTURA 14., 2002, Campo Grande, MS. Resumos.... Campo Grande: CONBRAPI, 2002. V.1 p.31. 